my longest laryngeal forceps, but which I have succeeded in reducing almost to a vanishing point by the use of the galvano-cautery bent down to as great a length. For such a small stump as $I$ have described the lightest touch is all that is desirable and ought indeed to be much less than at the time appears sufficient. In addition to the destruction of a portion by the actual burning away of substance the subjacent part of the growth undergoes a certain amount of shrinkage. Complete burning away of the growth would therefore be attended by a risk of undesirable cicatrisation of the normal underlying structures.

I do not wish it to be inferred that in these manipulations I have never touched the normal neighbouring parts of the larynx. In Case 1 I certainly did avoid doing so ; in Case 2 I more than once cauterised the superjacent portion of the ventricular band and also the opposite vocal cord. In the third case to which I have made reference the patient was exceptionally restless besides being absolutely intolerant of the "epiglottis-lifter" and I slightly cauterised the laryngeal surface of the epiglottis. As a rule I was able to confine the momentary cauterisation to the desired spotand in no instance did the involvement of the adj xcent parts mentioned occasion anything approaching obstructive cedema or indeed any symptom beyond a slight increase of hoarseness, which rapidly subsided.

With the precautions I have mentioned I believe that we have in the galvano-cautery an instrument which might be more frequently employed for the purpose under consideration than appears to be usual.

Cavendish-square, $\mathbf{W}$.

\section{A NON-TOXIC PREPARATION OF IODINE.}

By V. H. WYATT WINGRAVE, M.D. DURH., M.R.C.S. ENG.,

PHYSICIAN TO THE CENTRAL LONDON THROAT AND EAR HOSPITAL.

BEING especially interested in the supplemental treatment of enlarged glands and adenoids with iodine, about seven years ago I made several experiments with preparations of that drug with the object of finding one which could be given internally in large doses for considerable periods without causing symptoms of iodism while exercising its specific effects on ly mphatic enlargements. The late Mr. Martindale and the manager of Messrs. Morson and Sons were good enough to try several formulæ for me in their laboratories and finally succeeded in producing an easily-made compound in which iodine was loosely combined with an organic sub. stance, rendering it fit for ready absorption without causing any irritation of the digestive canal and affording a high physiological activity without any evidence of intolerance. This preparation I have used ever since both in hospital and private practice, and having watched the effects have come to the conclusion that it has fully confirmed my first estimate of its value.

The formula which I now employ has been perfected for me by Mr. W. H. Martindale, is easily prepared, and is very palatable. The iodine is in loose chemical combination with tannic acid, since none can be demonstrated as free, yet it is sufficiently free to be given up readily to the tissues after absorption and consequently there is no chance of any local intestinal or gastric irritation, so often associated with the administration of free iodine. Such combination is one of a nature similar to that of oxyhæmoglobin. Formula:-iodine, $2 \frac{1}{2}$ grammes; tanuic acid, 4 grammes; alcohol (90 par cent.), 38 cubic centimetres; syrup, q s. to 75 cuoic centimetres. The iodine is dissolved in the alcohol, the tannic acid and 30 cubic centimetres of the syrup are added, the solution is heated to just below boiling point until it affords no, evidence of free iodine with the starch reaction (about 20 minutes), is then cooled, and the remainder of the syrup is added with flavourings. Wxch drachm contains two grains of iodine. It may be given in doses of from half a drachm to two drachms in water or wine before meals, according to age.

I have found that children tolerate it well and it has proved specially useful in cases of chronic lymphadenitis associated with, or independent of, enlarged tonsils and adenoids, especially when the enlarged cervical glands persist after tonsillotomy. It is further indicated in those children who possess but slightly enlarged faucial and pharyngeal tonsils in whom operation is contra-indicated or objected to. In atrophic rhinitis it has afforded gratifying results especially when combined with arsenic; also in simple bronchocele supplemented with the use of iodine oleate (1 in 9 of oleic acid) as a pigment.

Sach an organic combination of iodine is by no means new as it was introduced many years ago in the form of a wine"Vin Nourry"-but so far as I know no definite or simple formula suitable for prescribing purposes has been available. It is for this reason and for the very satisfactory results which it has afforded in a prolonged trial that I venture to recommend it.

Devonshire-street, $\mathrm{w}$.

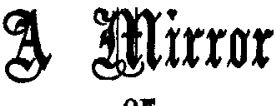

\section{HOSPITAL PRACTICE, BRITISH AND FOREIGN.}

Nulla autem est alia pro certo noscendi via, nisi quamplurimas et morborum et dissectionum historias, tum aliorum tum proprias collectas habere, et inter so comparare-MorgagnI De Sed. et Caus. Morb., lib. iv., Procemium.

\section{THE FRENCH HOSPITAL, SHAFTESBURY AVENUE.}

SIX CONSECUTIVE CASES OF DISEASE OF THE FALLOPIAN TUBES.

(Under the care of Dr. SEPTIMUS SunderLand and Mr. EDMUND OWEN.)

For the notes of the cases we are indebted to Mr. Jehan M. Barlet, resident medical officer.

OASE 1. Double tubo-ovarian abscess.-A married woman, aged 39 years, who had borne no children, was admitted into the French Hospital on Oct. 8th, 1903, for pain in each ovarian region. She had bad gonorrhoea and had been in the French Hospital two years previously with the same symptoms. On vaginal examination a semi-elastic swelling of about the size of a hen's egg could be felt on the right side, but nothing definite could be made out on the left, though she complained of more pain on that side.

$\mathrm{Mr}$. Owen made a three-inch incision in the median line and finding the right ovary and tube enlarged removed them, the tube rupturing under the manipulation and pus escaping. The soiled viscera were carefully wiped over but no irrigation was employed. The left ovary and tube were also diseased and were removed. A drainage-tube was left in for 48 hours. The woman made an uninterrupted recovery.

CASE 2. Double tubo-ovarian abscess.-A woman, aged 32 years, who had been married ten months and had had no children, was admitted into the French Hospital on Nov. 11th, 1903, for pain over each ovary. For six months previously she had had pain in the right iliac fossa, there being a history of a diminution of the menstrual flow for two months, followed by an advance of the period by 15 days, lasting 15 days. Her husband had had gonorrhoea.

A three-inch median incision was made. The left tube was much dilated and the ovary was enlarged; they were removed. The right tube and ovary were then brought to the surface and also found to be diseased and were likewise removed. (On opening the tubes subsequently pus was dis. covered in each.) A drainage-tube was left in for 48 hours. She also made an uninterrupted recovery.

CASE 3. Encysted purulent peritonitis secondary to pyo. salpinx.-A woman, aged 30 years, was admitted into the French Hospital on Jan. 20th, 1904 for abdominal pain. There was a large swelling in the right iliac fossa. There was a history of pains in the lower abdomen during six years; they had usually passed off with rest and douches. 12 days before admission she had been seized with severe pains and had passed a dark clot of blood per vaginam. She said that she had had a temperature going up to $103^{\circ}$ and $104^{\circ} \mathrm{F}$. and also that a large swelling had begun to form in the right lower abdomen. On admission she was looking ill; she vomited. The pulse was 124 and the temperature was $100^{\circ}$. The abdomen was distended, especially on the right side. There was a limitation of respiratory movements. On palpation a large swelling could be felt in the right iliac region, reaching 
across the middle line; fluctuation could be obscurely obtained. Percussion showed that intestine lay over the upper part of the tumour. On vaginal examination a hard mass was made out against the right fornix, the uterus being pushed over to the left. By bimanual examination fluctuation was obtainable; the mass was fixed.

Mr. Owen opened the abdomen on the day following her admission by a median incision below the umbilicus. The small intestines were congested, covered with lymph, and adherent to one another, surrounding a collection of foulsmelling pus. The abdominal cavity was washed out with abundant hot saline solution and two large tubes were brought out by counter-openings, but the case seemed hopeless. The source of the pus could not then be discovered as all the tissues were closely matted together. The patient was very much collapsed after the operation but by stimulation she recovered from the shock. She died early on the following morning.

Necropsy.-The contents of the pelvis were matted together by adhesions and between the rectum and the uterus was an abscess containing about ten ounces of pus which had not been discovered at the operation. There was pus in the right Fallopian tube and ovary, and in the body of the uterus was a large, deep ulcer which encroached upon the opening of the right Fallopian tube. At one spot the wall of the bladder was ulcerated, leaving only the mucous lining.

CASE 4. Ectopic gestation.-A woman, aged 28 years, who had had two children, was admitted into the French Hospital on Jan. 15th, 1904, for pains in the lower abdomen on the right side. Her history was as follows: On Christmas Day, when walking with her husband, she was suddenly taken faint and had to be carried home. From that time she had been constantly losing blood. The pain continued and she had neuralgia down the inside of the thigh. She frequently vomited. There was no history of gonorrhœa. The temperature was normal. Examination showed that there was acute tenderness over the region of the right ovary, a resistance being distinguishable. The uterus was moveable and the os was capable of admitting the tip of the index finger. Against the right fornix a tender, moveable, semi-elastic swelling, of about the size of a hen's egg, could be distinguished.

$\mathrm{Mr}$. Owen made a median incision, about four inches long, and introduced three fingers into the abdominal carity. A smooth, non-fluctuating swelling was made out on the right side, slightly adherent posteriorly. On bringing it to the surface it was found to be glistening and dark purple. The tube was ligated close to the uterus ; the broad ligament was tied off and the mass was removed. On cutting into it a large blood clot was found associated with a ruptured tubal gestation. There was also a small cyst of the ovary. The pelvis was wiped clean of blood and the wound was closed. A few weeks later she was sent to the convalescent home at Brighton.

CASE 5. Right pyosalpinx; left suppurating ovarian oyst.A woman, aged 27 years, married, with no children, was admitted into the French Hospital on Jan. 15th, 1904. Sbe had had no miscarriages. She complained of constant pain in the left iliac fossa. There had been no irregularity in menstruation except that during the last two months she had had three periods. A short time after marriage five years previously she had a thick yellow discharge associated with pains which had continued off and on during all these years. She had been persistently treated by douches and rest but without material advantage. Vaginal examination showed that the uterus was fixed and thrust backwards by an elongated mass on the left side, which was also fixed to the rectum and bulged against the left fornix. On the right side was a moveable, semi-elastic swelling of about the size and shape of a hen's egg and partly posterior to the uterus.

A median incision having been made, the right tube was found to be adherent to the surrounding tissues; these adhesions were broken through and the swollen and congested tube, thickly covered with recent lymph, was brought to the surface, tied off, and removed. The left tube was made out with difficulty, as it was concealed by, and was closely adherent to, the posterior surface of the uterus, the rectum, and the sigmoid flexure. These adhesions having at length been severed a mass of about the size of an orange was brought to the surface, secured, and removed. There was a good deal of oozing, the blood being mopped out with sponges soaked in a very hot saline solution. A Keith's tube was inserted and the abdominal wound was sewn up. The drain was removed after 48 hours, there being only a slight serous discharge obtained from it. The temperature remained normal and the pulse $n \in v e r$ rose above 84 per minute. On examining the masses removed the one on the right side proved to be a pyosalpinx, whilst the one on the left side had the appearance of an ovarian cyst which had suppurated. She made a rapid recovery.

OASE 6. Right salpingitis and oyst in the broad ligament; left salpingitis.-The patient, a widow, aged 24 years, was admitted into the French Hospital on Feb. 1st, 1904, for great pain in the right iliac fossa. She had had no children but had one miscarriage four years before. A week previously she had been wrestling and on the next day she felt an acute stabbing pain in the right iliac fossa, which doubled her up and, as she says, felt like a bearing-down pain. At the time of her violent effort she was in the middle of $h \in \mathrm{r}$ monthly period, and after the attack of pain the flow increased very considerably, with the passage of clots. She had always been regular. The abdomen moved fairly well with respiration, except in the lower segment, where there was a slight resistance on the right side. Vaginal examination showed that the os was patulous and that the uterus was definitely enlarged and pushed over to the left side by a semi-elastic swelling which bulged down against the right fornix. It seemed to be of about the size of a small orange. Above this could be felt a distended tube. There was free discharge of blood from the uterus. Dr. Sunderland had seen her in consultation at her own house and, recognising the presence of an acute pelvic condition which evidently needed exploration, sent her into the hospital for operation. This was performed on the following day. Colonel C. H. Joubert, I.M.S., late professor of obstetrics at the Calcutta Medical College, was present at this operation.

A median incision having been made, there was found on the right side a dark and adherent mass which proved to be the distended Fallopian tube; below this a cyst was found as big as a small orange lying between the layers of the broad ligament in close connexion with the enlarged Fallopian tube. The cyst burst on being freed from adhesions, discharging a thin sanguineous fluid. The whole mass was tied off in sections and removed. On the left side a slightly enlarged tube showing evidence of salpingitis was found behind the uterus and removed. The oozing from the detached adhesions was stopped with sponges wrung out in hot saline soluticn. A Keith's tube was left in for 24 hours. The mass from the right side, which had appearances suggestive of an extra-uterine gestation, was sent to Dr. G, Leslie Eastes for examination who reported as follows: "This specimen shows the presence of thrombosed vessels, and free hæmorrhages in the walls of the tube, together with a large amount of granulation tissue. Chorionic villi have not been found, so that tubal gestation cannot be diagnosed. We must conclude that the intramural hæmorrhage was due to some acute condition supervening upon a chronic inflammatory process." The patient, who strenuously denied the possibility of ectopic gestation, made an uninterrapted recovery.

Remarks by Dr. SUNDERLAND and Mr. OWEN.-The misapprehension that gonorrhoa is not a very zerious disease is widely spread. It has been argued that though it is a common affection of young adults one rarely hears of a man being permanently damaged by it, provided only he has escaped being the subject of organic stricture of the urethra and the various diseases of the bladder and kidneys which may depend upon it. But the surgeon who sees a patient with secondary gonococcal inflammation of the knee-joint or of a series of joints, and the physician who is called to advise in the treatment of a case in which a pyæmic affection of the pericardium has followed in the wake of a venereal urethritis, cannot fail to regard the primary disease as one of extreme potential gravity. But perbaps the saddest, as well as the most serious, of the complications of gonorrhœa are met with in the case of the married woman whose life is rendered miserable and uncertain by infection which was unwittingly communicated to her by her husband. The probability is that the man honestly believed himself to be, and was duly advised that he was, perfectly cured of his urethritis at the time of his marriage, when, as a matter of fact, gonococci were still present in the urethral canal. It is quite possible, moreover, that the wife was infected with the micro-organisms without realising that there was anything seriously amiss with her, that the area of incubation of the micro-organisms was chiefly confined to the interior of the cervix uteri, for instance, and that she had none of the usual symptoms of 
gonorrhœal infection. It not infrequently happens that when the practitioner has asked all the questions which he deems it prudent to put to the wife, he leaves off his crossexamination without having obtained collateral evidence of her having been definitely infected, though the confessions of the husband and the circumstances of the case leave little room for doubt.

Of the four cases of pyosalpinx reported above, Nos. 1 and 5 gave a clear history of gonorrhoeal infection; No. 3 had probably been a victim to it, and No. 2 might well have been infected from her husband. No. 3 is one of those sad cases in which infection spreads from a tubal abscess to involve the peritoneum in a fatal suppuration. Bacilli coli communes must have been present in myriads, the original tubal swelling having been probably infected from adherent intestine. The history of this case adds further support to the teaching of those who advise that in all cases in which the existence of a tubal abscess is suspected the tumour should be explored without delay by abdominal section, and then dealt with in the only possible way-that is, of course, by removal.

Another point for remark is that if in the process of being separated from the surrounding adhesions the cyst gives way and its foul contents spread themselves over the adjacent coils of intestine, the most practical manner of cleansing these is by the free use of dry absorbent sterilised swabs. Formerly it was very much the custom to deal with the soiled viscera by hot flushings, but though in cases where the pus was not sterile this method could not be depended upon to wash away all the germs it was certain to convey some of them to parts of the serous membrane which, up to that time, they had not reached. In operations in which the foul contents of an appendicular or a tubal abscess unavoidably find access to the serous covering of neighbour ing viscera, is not the simple process of wiping the part clean with a soft dry sterilised swab as likely to prove efficient as the less convenient one of irrigation? In every case the surgeon should do his best to prevent the occurrence of any fouling, but if, notwithstanding, it does occur and the case turns out successful nevertheless, he must not ascribe all the credit for the happy result to the way in which he dealt with the straying pus.

What is the explanation of the farourable course which cases often run when the surgeon has concluded his opera. tion with the full knowledge that pus has been left bebind? It is possible that the escaping pus was sterile. In other cases where pus which contained bacilli escaped upon and contaminated the peritoneum and no worse results followed than a slight localised peritonitis, it is probable that the formation of leucocytes and plasma-cells on the peritoneal surface prevented the entrance of the poison to the system. Possibly, also, during the time that an abscess was forming an antitoxin was being prepared in the blood which sufficed to render harmless the occurrence of, or even to prevent, a localised peritonitis. Therefore, when there is no urgent indication for immediate operation it is advisable to prepare the patient for the operation by rest in bed, good feeding, and a course of ferruginous tonics so that the blood cells may be the better able to resist the ravages which might be caused by bacilli-laden pus if the tubes happen to rupture during the operation.

In $1899 \mathrm{Mr}$. A. G. R. Foulerton reported the results of 459 cases of salpingitis (including 16 cases investigated by himself) in which the pus had been examined by competent observers. In about 20 per cent. of these cases the gonococcus was discovered, in about 20 per cent. bacteria other than the gonococci were found (including the staphylococcus pyogenes albus, the bacillus coli communis, the bacillus tuber culosis, and the streptococcus pyogenes), but in the remaining 60 per cent. the contents of the tubes had been found absolutely sterile. He explained this absence of bacteria by the fact that many cases of pyosalpinx are not operated upon till a considerable period after the formation of the abscess, by which time the causative parasites have perished as the result of starvation or of being poisoned in a small closed cavity by their own excretory products. With regard to the 20 per cent. of cases in which other bacteria than gonococci had been found, in a certain proportion of them the bacillus discovered did not always harmonise with the etiology of the disease, for in some of them the pus had become sterile and had been secondarily infected with the bacillus coli communis from adherent intestine. It is thus impossible to determine frum examination of the pus contained in the tubes the proportion of cases which owe their origin to gonorrhcea. But it is our opinion, judging partly from the records of $\mathrm{Mr}$. Foulerton and partly from our own clinical experience, that the number of cases of pyosalpinx which owe their origin to gonorrhœa is considerably under-estimated and that the proportion given by text-books of a percentage of from 20 to 30 is far below the mark.

In a case of salpingitis arising during an attack of gonor rhoea when there is evidence that pus has formed in one or in both tubes the questions arise : Should the condition be dealt with per vaginam (through Douglas's pouch), as recommended by some surgeons, or by laparotomy? And, secondly, when should operation be undertaken? In our opinion cases in which incision and drainage of the tube through the vagina should be resorted to must be of very rare occurrence. When there are much matting and adhesion it must be difficult to insure that all the pus has been evacuated, and certainly it seems a more sound principle to remove the whole of the diseased tube by laparotomy, as in the case of intrapelvic abscess due to disease of the appendix creci. With regard to the time for operation, it is probably wise to allow the patient to get over the acute inflammatory attack and unless urgent symptoms should call for interference to wait for a few months until the virulence of the contents of the tubal abscess may have abated in order to minimise the risk of infecting the peritoneum. It is impossible to lay down definite rules, for the circum. stances of each case have to be considered. Indeed, some of these cases if left alone get well without operation, the pus apparently becoming absorbed.

In conclusion, we would like to insist upon the importance of the careful and thorough treatment of gonorrhoea in females in its initial stage. This cannot be too strongly put forward when one considers the amount of suffering and the risk to life which may attend upon an apparently slight attack of the disease.

\section{attedical Soxtetixs.}

Society for the STUdy of Disease IN ChILDRen.-A meeting of this society was held on March 18th, Dr. Percy G. Lewis being in the chair.-Mr. Harold Burrows showed a case of Enlarged Liver and Spleen in a girl, aged 13 years. Six months previously laparotomy had been performed for supposed hydatid disease of the liver but the liver was found to be. uniformly enlarged and smooth. There was no history of syphilis but as there were the remains of old retino-choroiditis in both eyes a diagnosis of syphilis had been made.-Dr. C. W. Chapman showed a girl, aged 12 years, who had suffered from Mitral Disease with recurrent attacks of Dilatation of the Heart. These had at first yielded to the ordinary remedies but later the dilatation and failure of compensation which were accompanied by crthopncea were not relieved until solution of adrenalin was administered in five-drop doses every four hours. This was followed by steady improvement.- $\mathbf{M r}$. J. Keogh Murphy showed a case of Hæmatoma of the Parietal Bone in an infant, aged eight weeks, and a case of Double Pes Cavus in a boy, aged four years.-Dr. Leonard G. Guthrie showed a boy, aged 11 years, who for two years had beer. subject to peculiar attacks in which he would suddenly run to his mother, exclaiming, "Horrid smell." He would appear much agitated and distressed for a few moments, sometimes spitting and grimacing, and going through the action of trying to pull something out of his mouth. He would then behave "as if he were silly," running to persons in the room and trying to embrace them. After five or ten minutes of this behaviour he would become quiet, turn pale, shiver as if cold, seem exhausted, and lie down to sleep. Dr. Guthrie regarded the case as one of automatism with olfactory and gustatory aure, a condition allied to epilepsy or megrim. Under treatment with bromide of potassium in eight grain doses thrice daily the attacks diminished rapidly in frequency and severity.-Dr. Lewis commented on the large pupils and a tendency to exophthalmos in the patient.-Dr. E. A. Jones said that visual auræ were of two kinds, either of persons which were usually unpleasant, or of landscapes which were usually pleasant.Dr. Edmund Cautley showed a case of Multiple Congenital 